\title{
BMJ How does a history of psychiatric OPen hospital care influence access to coronary care: a cohort study
}

\author{
Kristiina Manderbacka, ${ }^{1}$ Martti Arffman, ${ }^{1}$ Reijo Sund, ${ }^{1}$ Jari Haukka, ${ }^{1,2}$ \\ IImo Keskimäki, ${ }^{1}$ Kristian Wahlbeck ${ }^{1}$
}

To cite: Manderbacka K, Arffman M, Sund R, et al. How does a history of psychiatric hospital care influence access to coronary care: a cohort study. BMJ Open 2012;2:e000831.

doi:10.1136/

bmjopen-2012-000831

- Prepublication history for this paper is available online. To view this file please visit the journal online (http://dx. doi.org/10.1136/ bmjopen-2012-000831).

Received 10 January 2012 Accepted 7 March 2012

This final article is available for use under the terms of the Creative Commons Attribution Non-Commercial 2.0 Licence; see http://bmjopen.bmj.com

${ }^{1}$ National Institute for Health and Welfare, Helsinki, Finland ${ }^{2}$ Department of Public Health, Hjelt Institute, University of Helsinki, Helsinki, Finland

Correspondence to Dr Kristiina Manderbacka; kristiina.manderbacka@thl.fi

\section{ABSTRACT}

Objectives: Equity in physical health of patients with severe mental disorders is a major public health concern. The aim of this cohort study was to examine equity in access to coronary care among persons with a history of severe mental disorder in 1998-2009.

Design: Nationwide register linkage cohort study. Setting: Hospital care in the Finnish healthcare system.

Population: The study population consisted of all residents in Finland aged 40 years or older. All hospital discharges in 1998-2009 with a diagnosis of coronary heart disease or severe mental disorder were obtained from the Care Register.

Primary outcome measures: Data on deaths, hospitalisations and coronary revascularisations were linked to the data set using unique personal identifiers.

Results: Patients with severe mental disorders had increased likelihood of hospital care due to coronary heart disease (RR between $1.22,95 \% \mathrm{Cl} 1.18$ to 1.25 and $1.93,1.84$ to 2.03 in different age groups) and in 40-49-year-olds also increased likelihood of revascularisation $(1.26,1.16$ to 1.38$)$ compared with persons without mental disorders. Access to revascularisation was poorer among older persons with severe mental disorders in relation to need suggested by increased coronary mortality. In spite of excess coronary mortality (ranging from $0.95,0.89$ to 1.01 to $3.16,2.82$ to 3.54 ), worst off were people with a history of psychosis, who did not have increased use of hospital care and had lower likelihood of receiving revascularisations (ranging from $0.44,0.37$ to 0.51 to $0.74,0.59$ to 0.93 ) compared with persons without mental disorders.

Conclusions: Selective mechanisms seem to be at work in access to care and revascularisations among people with severe mental disorders. Healthcare professionals need to be aware of the need for targeted measures to address challenges in provision of somatic care among people with severe mental health problems, especially among people with psychoses and old people.

\section{INTRODUCTION}

Equity in health and equal access to healthcare according to need among vulnerable groups, such as people with severe mental

\section{ARTICLE SUMMARY}

\section{Article focus}

- Equity in physical health of patients with severe mental disorders is a major public health concern since increased morbidity and mortality due to somatic disorders has repeatedly been reported among them.

- The aim of this cohort study was to examine equity in access to coronary care among persons with a history of severe mental disorder in Finland in 1998-2009.

\section{Key messages}

- Our results show poor access to coronary revascularisation according to need among people with severe mental disorders and especially poor access to hospital care and revascularisations among people with psychotic disorders.

- Healthcare professionals need to be aware of the need for targeted measures to address challenges in provision of somatic care among people with severe mental health problems, especially among people with psychoses and old people.

Strengths and limitations of this study

- We used nationwide, unselective representative data concerning the total population of Finnish residents in 1998-2009 collected from administrative registers with full coverage and based on clinical diagnoses, which allowed us to put the outcomes in proportion to the population at risk.

- We were able to examine the three major diagnostic groups of severe mental disorders, namely mood disorders, psychotic disorders and substance abuse disorders.

- Our data do not enable us to evaluate whether the poor access to care according to need among people with severe mental disorders is due to delay or avoidance in seeking care or poorer responsiveness of the healthcare system to somatic health problems of these vulnerable patient groups.

disorders, is an indicator of well-functioning health systems. Nevertheless, increased morbidity and mortality due to somatic 
disorders, including cardiovascular disease, respiratory disease and cancer, has repeatedly been reported in persons with severe mental disorders compared with the total population. ${ }^{1-8}$ These findings may derive both from increased incidence and higher case death among patients with mental disorders. ${ }^{9}{ }^{10}$ Unhealthier lifestyles, poorer socioeconomic circumstances, adverse metabolic effects of antipsychotic medications and social consequences of mental illness such as social isolation are likely to explain part of the excess mortality. Another important factor likely to contribute to these differences is poorer access to and quality of healthcare. ${ }^{11} 12$

Accordingly, earlier studies have found elevated coronary heart disease (CHD) mortality but reported no or only slightly elevated use of hospital in- or outpatient care compared with other coronary patients. ${ }^{9} 13$ Studies have further reported that persons with a history of psychoactive substance use disorders, psychosis or mood disorders are less likely to receive $\beta$ blockers, aspirin, ACE inhibitors and statins compared with other coronary patients, ${ }^{14-18}$ to be less likely to have had a recent check-up of their cholesterol or blood pressure level $^{1519}$ and to undergo revascularisation operations less often. ${ }^{9131420}$ Similar results concerning coronary revascularisations have been reported even after controlling for cardiac risk factors and admission characteristics. ${ }^{21}$ The study results suggest that access to coronary care is not realised in proportion to need among persons with a history of psychiatric disorders. The studies have mainly been conducted in the USA and many of them have been based on small or regional samples or clinical samples of CHD patients. This study contributes with a nationwide analysis of access to coronary care and mortality, covering the total population, providing an opportunity to study and compare outcomes in all major diagnostic groups of severe mental disorders, including substance use disorders. The Finnish healthcare system is a good case for examining coronary care in a universal healthcare state since the Finnish system is funded mainly by tax revenues and based on universal access to care according to clinical need for all residents. ${ }^{22}$

The aim of this study was to provide a comprehensive analysis of equity in access to coronary care among people with a history of severe mental disorder. We examined coronary mortality, risk of hospitalisation and revascularisation procedures during the last decade (1998-2009). Three groups of patients with severe mental disorders were examined, that is, patients with psychoactive substance use disorders, psychotic disorders and mood disorders, and compared with population without hospitalisations due to psychiatric diagnoses. We further analysed the effects of sex and age to these differences.

\section{METHODS}

This nationwide register-based study examines the access to coronary care in 1998-2009. The study population consists of all men and women resident in Finland and older than 40 years at the beginning of each calendar year. All hospital discharges in 1998-2009 containing a diagnosis for CHD (ICD-10: I20-I25, I46.1, I46.9) or severe mental disorder (ICD-10: F10-F39) were obtained from the Finnish Care Register. The Care Register is maintained by the National Institute for Health and Welfare and includes data for all hospital inpatient care in Finland, both public and private. The register includes the dates for the beginning and end of each inpatient care episode, the main diagnosis for the episode (ICD-10) and two side diagnoses, as well as all surgical procedures.

The cohort with severe mental disorders was defined as those having a hospital care episode at least once with main diagnosis for a psychotic disorder (ICD-10 F20F29), mood disorder (ICD-10 F30-F39) or substance use disorder (ICD-10 F10-F19) at any time during 1 January 1998 to 31 December 2009. If an individual had been hospitalised more than once and given more than one main diagnosis, we hierarchically allocated the individual to the psychosis subpopulation if diagnosed with psychosis at least once. Patients with main substance use related disorders and mood disorder diagnoses were allocated to the substance use group. These persons remained in the population with mental disorders throughout the study period because of the long natural course of the mental disorders in question. CHD hospitalisations and coronary revascularisations among them were compared with those of the total population without severe mental disorders. The psychiatric population at risk was defined as the annual mean population of the severe mental disorder cohort in each year in 1998-2009. Stratification by year, 10-year age band, sex and patient group was used. Annual mean population figures for the whole Finnish population in 1998-2009 were obtained from Statistics Finland from which numbers of people with severe mental disorders were subtracted to achieve the population at risk for the nonpsychiatric population. Both were followed up from 1 January 1998 or the subsequent year following their 40th birthday until the end of the study period in 31 December 2009 or death. The age of the persons with and without mental disorders was updated in the end of each year.

The psychiatric population comprises some 1.4 million person-years including 579478 for substance abuse disorders, 399909 for psychotic disorders and 440752 for mood disorders, whereas the non-psychiatric population comprise some 27.5 million person-years over the study period. Data on coronary revascularisation were individually extracted from the register. Data on mortality were obtained from the cause of death statistics. ${ }^{23}$ These individual-level data sets were linked using unique personal identifiers.

The Finnish registers have been found to be a valid and reliable tool for epidemiological research. ${ }^{24-26}$ The Finnish Care Register has shown good validity with 
regard to psychotic disorders in general $^{27}$ and even in regard to a range of specific main mental health diagnoses, such as bipolar disorder, ${ }^{28}$ and alcohol use disorders. ${ }^{29}$

Main outcomes were RR of at least one hospitalisation for CHD, first coronary revascularisations (coronary artery bypass grafting or coronary angioplasty) and CHD mortality. Incidence rates and their 95\% CIs were calculated for each patient group by sex and age.

Poisson regression models were used to study differences between the patient groups in access to coronary care. The three outcome measures in relation to personyears were examined by patient sex, calendar year, age group, diagnostic group and the interaction between the last two. As interactions between the diagnostic groups and age groups were found, the RRs were calculated to assess differences between diagnostic groups in each age group. For the total risk ratios, the analyses were performed controlling for patient sex, calendar year, age group and diagnostic group. The analyses were performed using SAS V.9.2.

The need for revascularisation operations was assessed using two proxies. We compared likelihood of revascularisation against age-specific coronary mortality and risk of coronary hospitalisations in the three groups of persons with severe mental disorders against persons without these disorders. Ten-year age categories were used in plotting the death rates and risks of hospitalisation against the operation rates. The study protocol has been approved by the Research Ethics Committee of the National Centre for Health and Welfare (§163/2010).

\section{RESULTS}

There were 164999 people with severe mental disorders in the data during the study period of whom 67659 were classified as having a psychosis, 47205 as having a substance use-related disorder and 50135 as having a mood disorder. Those having psychotic disorders had 4991 coronary hospitalisations and underwent 568 revascularisations, those having substance abuse disorders had 8178 hospitalisations and 1823 revascularisations and those with mood disorders had 9629 hospitalisations and 1327 revascularisations. Persons without severe mental disorders had 370452 coronary hospitalisations and underwent 91996 revascularisations during the study period. Table 1 presents rates for hospitalisation and revascularisation per 100000 for each group of people with severe mental disorder by age and sex. Both hospitalisations and revascularisations were more common among men in each age group but the associations by different diagnostic groups were similar for men and women. Persons with psychotic disorder had lower rates for both hospitalisation and revascularisation compared with other groups with severe mental disorders, whereas the rates were higher among persons with substance use disorders and mood disorders compared with coronary people without mental disorders.
CHD mortality was higher among persons with severe mental disorders compared with people without a history of a severe mental disorder (table 2). The differences in mortality risk were especially marked among persons with disorders due to psychoactive substance use and psychotic disorders, and a pattern of decreasing excess risk was found by age: the younger the age group, the larger the excess mortality risk.

Overall, people with severe mental disorders had an increased risk of being hospitalised due to CHD compared with patients without mental disorders (table 3). This was especially true for younger (40-49 years old) persons with substance use disorders or mood disorders, among whom the likelihood of hospitalisation was more than double compared with those without mental disorders. A statistically significant age pattern was detected among these two patient groups: the younger the age group, the larger the excess likelihood of hospitalisation. In persons with a psychosis diagnosis, however, the likelihood was similar to those without mental disorders and no age gradient was detected.

The sex-specific analyses revealed that differences were more pronounced among women with disorders due to psychoactive substance use and mood disorders (data not shown).

The likelihood of coronary revascularisations (table 4) was higher among 40-49-year-old persons with disorders due to psychoactive substance use and 40-59-year-old persons with mood disorders. In older age groups among these patient groups and among persons with psychosis, the likelihood of revascularisation was smaller compared with persons without mental disorders. The likelihood of revascularisation was especially low among older $(60+$ years $)$ persons with psychotic disorders among whom the RR was approximately half of that among persons without mental disorders. We further analysed whether the results differ by type of first revascularisation (bypass operation, angioplasty). Since the results were similar for both types of revascularisation, numerical results are not presented.

Age-specific revascularisation rates are plotted against age-specific coronary mortality rates and hospitalisation rates in figure 1 . Among persons with substance use disorders, revascularisation rates were below those found among persons without mental disorders at each level of mortality except for the two oldest age groups (figure 1A). Among persons with mood disorders, revascularisation rates exceeded than those found among persons without mental disorders in the youngest age group but were lower in older age groups at each level of mortality. Among persons with psychotic disorders, revascularisation rates were below than those found in other groups at each level of coronary mortality. When coronary hospitalisation was used as a proxy for need (figure 1B), the curves were similar among persons with substance use disorders and mood disorders: at each level of hospitalisation, the revascularisation rates were lower than those among persons without mental 


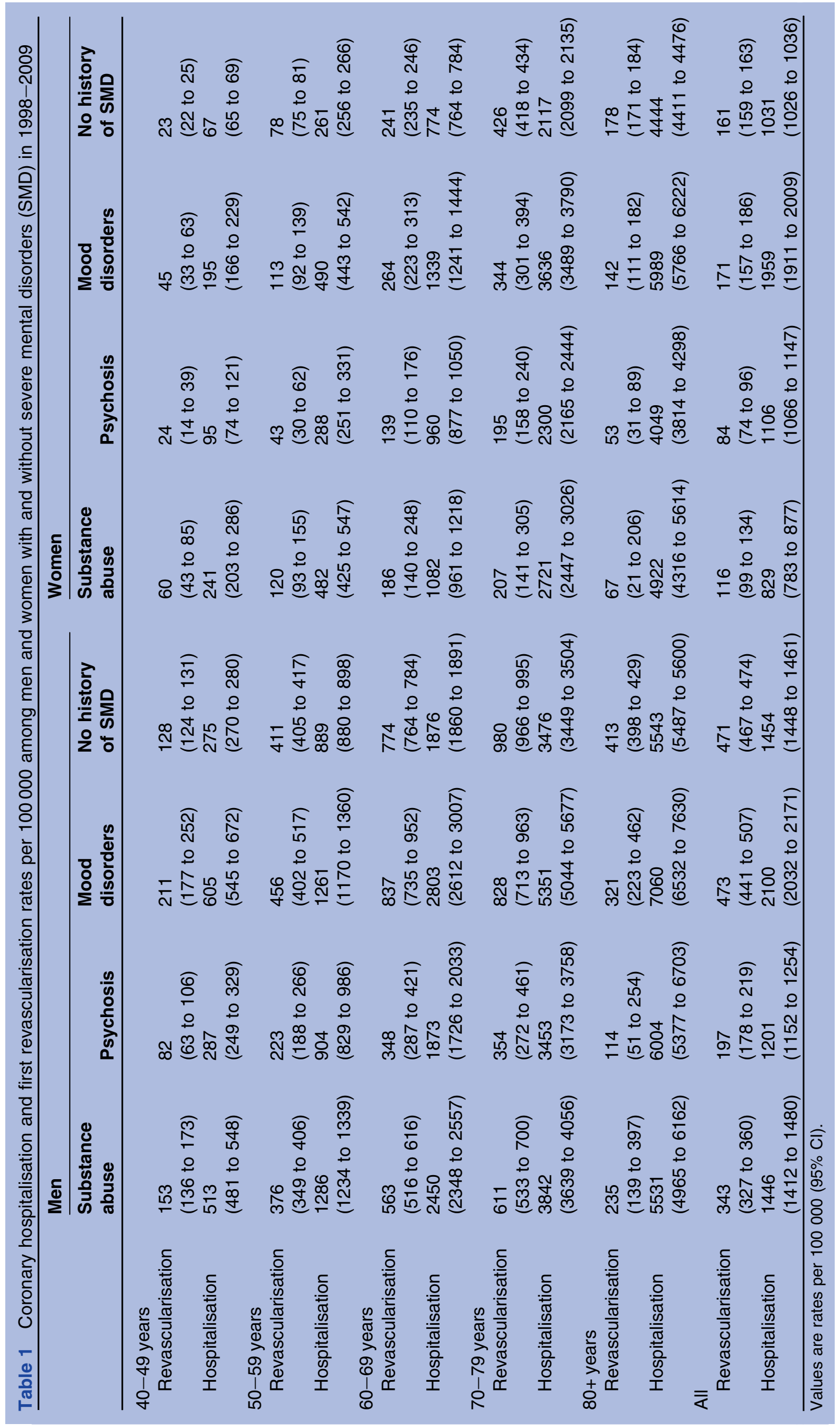



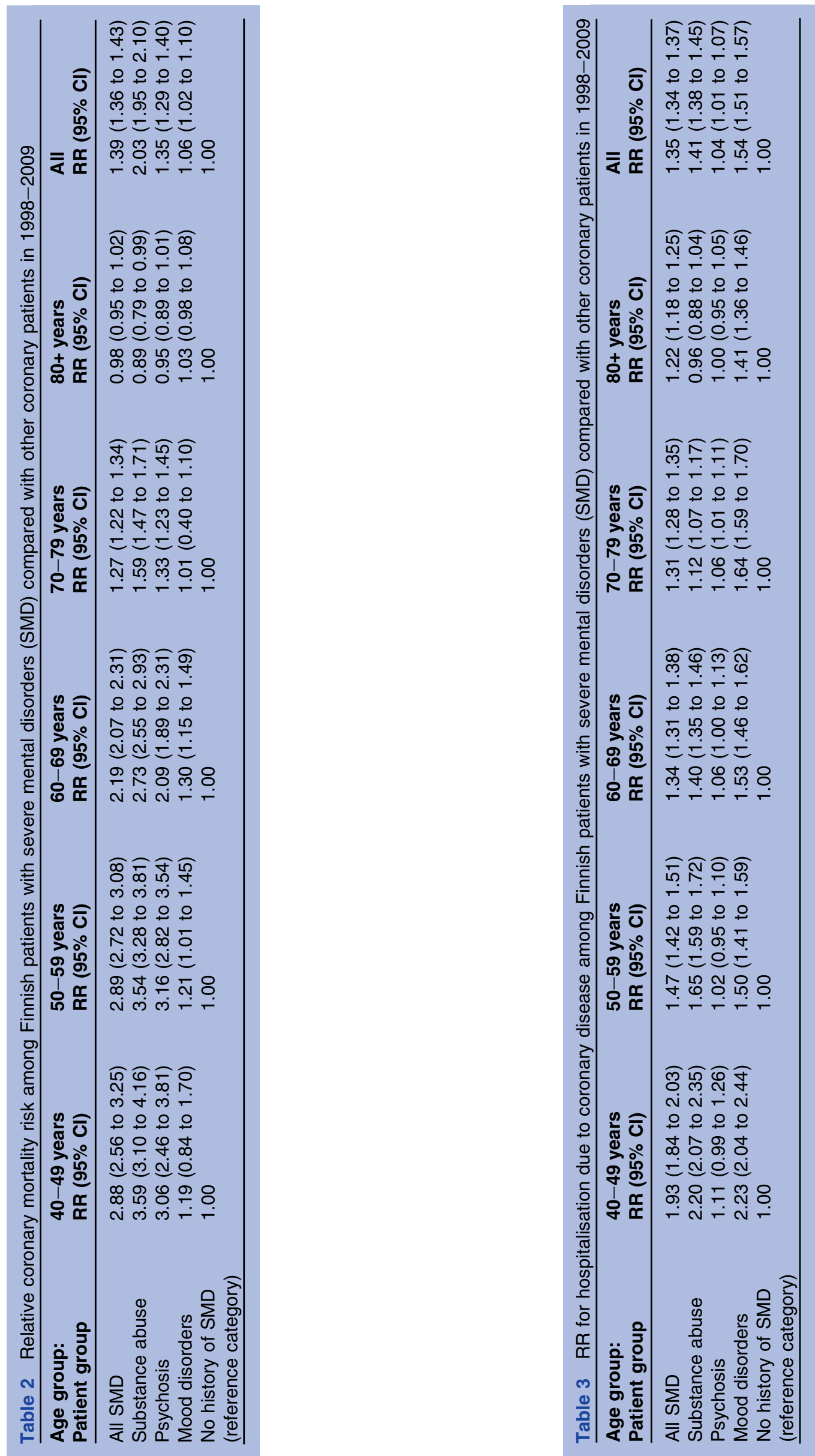


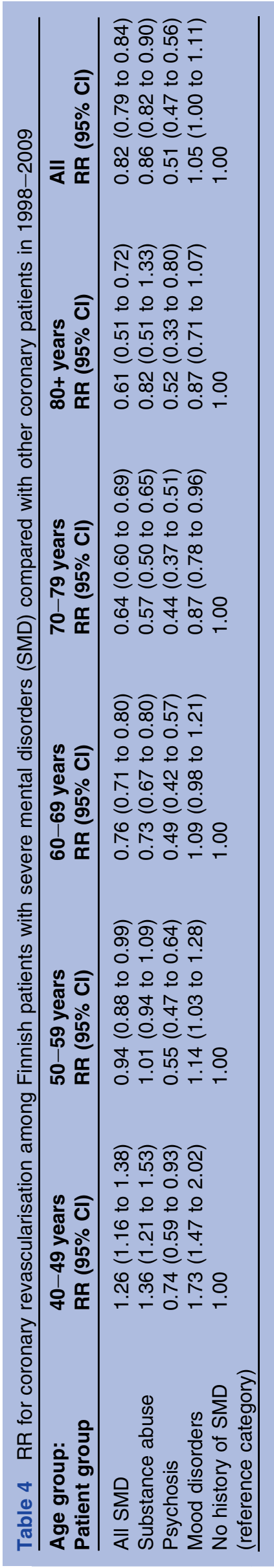

disorders except for the oldest age group. The rates were especially low among persons with psychotic disorders.

\section{DISCUSSION}

This nationwide register-based study examined equity in coronary care among the three major groups of people with severe mental disorders in 1998-2009 in Finland compared with people without these disorders. Three main outcomes were examined, namely coronary mortality, coronary hospitalisation and first coronary revascularisations. Persons with substance use disorders or mood disorders had increased likelihood of hospital care due to CHD but not persons with psychosis. The same pattern was seen for coronary revascularisations among young age groups. Increased hospitalisations and revascularisations are likely to reflect the increased prevalence of CHD among people with severe mental disorders as suggested by increased coronary mortality reported in earlier studies. ${ }^{1-8}$ However, access to revascularisation was poorer among older persons in these patient groups in relation to need suggested by increased CHD mortality indicating that selective mechanisms may differ across age groups. Whereas earlier research has shown increased CHD incidence and mortality among persons with psychotic disorders, ${ }^{5}$ corroborated by our results of increased coronary mortality, persons with psychosis had no differences in the likelihood of hospital care to persons without severe mental disorders. We further found selective underrepresentation of people with psychotic disorders among those receiving revascularisation interventions in all age groups. While older persons with substance use disorders and mood disorders had poorer access to revascularisation compared with those without severe mental disorders, the risk ratios were especially low among persons with psychotic disorders. Selective mechanisms seem to be at work at two levels in this patient group. First, these patients seem to have poorer access to hospital care as compared with people with mood or substance abuse-related disorders and second, poorer access to revascularisation.

The strength of our study was that we used nationwide, unselective representative data concerning the total population of Finnish residents in 1998-2009 collected from administrative registers with full coverage and based on clinical diagnoses. We were also able to include persons with substance use disorders. The accuracy and coverage of the Finnish Care Register has, in general, been reported to be good, ${ }^{23}$ and the accuracy of $\mathrm{CHD}$ data in the register has proved to be good. ${ }^{25} \mathrm{~A}$ weakness of our study is that persons with less severe mental disorders, who have not been hospitalised, are excluded from the population with severe mental disorders and can therefore appear in the population without severe mental disorders. Thus, our results are representative only for the population with mental disorders severe enough to require hospitalisation and rather underestimate the differences. Another weakness of our study is 
A Revascularisations per 100000 at risk

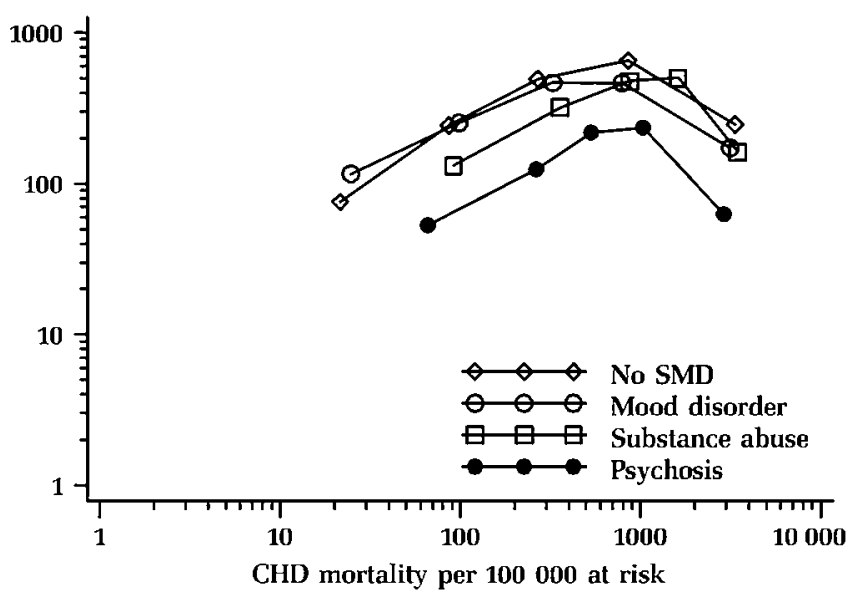

B Revascularisations per 100000 at risk

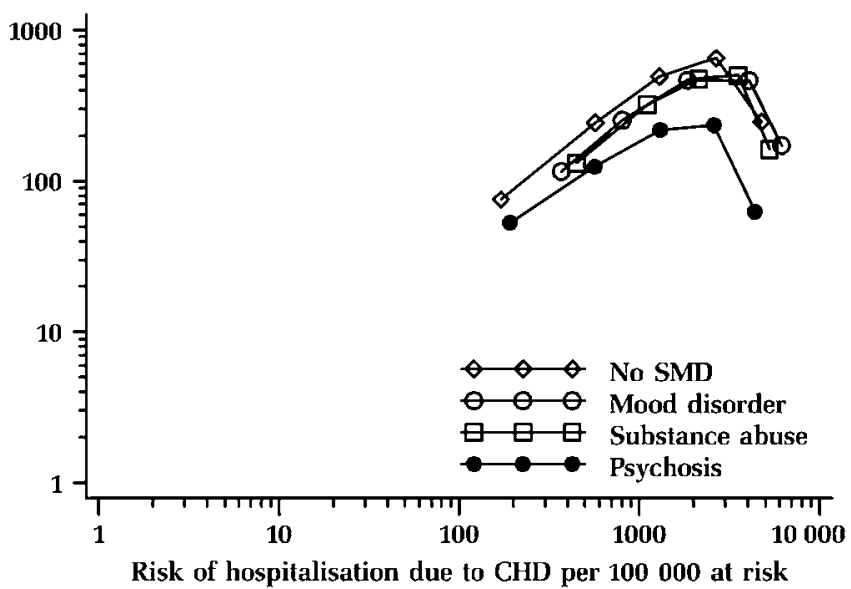

Figure 1 The effect of severe mental disorders on the rates of coronary revascularisation and coronary heart disease (CHD) mortality (A) and coronary hospitalisation (B) (each point represents 10-year age band across the age groups 40+ years, logarithmic scale). SMD, severe mental disorders.

that we could not control for possible comorbidities or health behaviours that could have an effect on the differences.

Our results concerning coronary mortality, hospitalisations and revascularisation procedures are in line with those reported from studies examining total populations of persons with specific severe mental disorders ${ }^{1} 91314$ and our results replicate especially the low hospital admission rates and revascularisation rates among persons with psychosis in relation to need indicated by excess coronary mortality. ${ }^{16}$ Some studies have reported no differences in hospitalisations or revascularisations, but these studies have been based on clinical coronary populations and not total populations of persons with severe mental disorders, ${ }^{30-32}$ whereas our study covered the total population of persons with mental disorders severe enough to require hospitalisation. Our study design allowed us to put the outcomes in proportion to the population at risk. Our study further adds to the literature by examining the outcomes by age. Our results suggest that both access to and quality of care as well as outcomes may differ by age among persons with severe mental disorders. Additionally, studies including substance use disorders are rare. $^{13} 33$ Kisely and colleagues $^{33}$ reported similar likelihood of coronary procedures among persons with substance use disorders compared with the total population from Nova Scotia, Canada. Lawrence and colleagues ${ }^{13}$ reported similar admission rates, and among men similar revascularisation rates, among persons with substance use problems compared with the general population in Western Australia.

The register data used in our study do not enable us to evaluate whether the poor access to coronary revascularisation according to need found in our study among people with severe mental disorders and especially poor access to hospital care and revascularisations among people with psychotic disorders are due to delay or avoidance in seeking care or poorer responsiveness of the healthcare system to somatic health problems of these vulnerable patient groups. Both may be related to stigma associated with mental health disorders and especially with psychosis. ${ }^{34}$ Another possible explanation that can play a role in differences in revascularisation rates are ethical and consent issues as well as contraindications for invasive surgical procedures due to comorbid conditions or treatments. The age gradient in access to CHD treatment points towards a role of stigma, as stigmatising attitudes towards mental disorders are more common among older people in Finland. ${ }^{35}$ The lack of access to coronary treatment is likely to reflect shortcomings in provision of health services and in care seeking because of stigma associated to mental disorders in healthcare and self-stigma among people with mental disorders interact. Those already labelled by the health services may decide to distance themselves from the label, forgoing treatment or becoming noncompliant. ${ }^{36}$ Our results are in line with the high level of discrimination experienced especially by people with schizophrenia. ${ }^{37}$

Our results suggest that even in a highly developed welfare state with an egalitarian public health system with full population coverage, intentional or unintentional structural discrimination of people with severe mental disorders, especially people with psychotic disorders, occurs. The healthcare professionals need to be aware of possible stigmatising attitudes among them and of the need for targeted measures to address the needs for somatic healthcare among people with severe mental health problems and especially among people with psychosis. Addressing stigma is a core component in aspirations to reduce excess cardiovascular mortality among people with severe mental disorders.

Contributors KM participated in the study conception and design, drafted the manuscript, participated in review and interpretation of the results and revision 
of the manuscript and has approved the final version. She is the guarantor of the manuscript. MA participated in the study conception and design, performed the data preparation and analysis and participated in drafting of the methods and findings sections of the manuscript, participated in review and interpretation of the results and has seen and approved the final version. RS participated in the study conception and design, guided the data analysis, participated in the interpretation of the results and preparation of the manuscript and in review and interpretation of the results and has seen and approved the final version. JH participated in the study conception and design, participated in the interpretation of the results and drafting of the manuscript and in the review and interpretation of results and has seen and approved the final version. IK participated in the study conception and design, participated in the interpretation of the results and drafting of the manuscript and in the review and interpretation of results and has seen and approved the final version. KW participated in the study conception and design, oversaw the data analysis, design and drafting of the manuscript and participated in the interpretation of the results and in the review and interpretation of results and has seen and approved the final version. He is the guarantor of the manuscript.

Funding This work was partly supported by the Academy of Finland (project number 133793) but it had no involvement in its design, data collection, findings or decision to publish.

Competing interests $\mathrm{JH}$ has been in research collaboration with Janssen-Cilag, Eli Lilly, Novartis, Novo Nordisk, Astra-Zeneca, Sanofi-Aventis and Nycomed and have been a member of the expert advisory group for Astellas. IK has received funding from the Academy of Finland for the study but the Academy had no involvement in its design, data collection, findings or decision to publish and is asked to advise the Finnish Ministry of Health and Social Affairs from time to time on matters relating to socioeconomic inequality and health: regardless of the findings of this study, the outputs of this research would form part of that advice. KM, RS, MA and KW declared no conflict of interest.

Ethics approval Ethics approval was provided by the Research Ethics Committee of the National Centre for Health and Welfare (\$163/2010)

Provenance and peer review Not commissioned; externally peer reviewed.

Data sharing statement No additional data available.

\section{REFERENCES}

1. Laursen TM, Munk-Olsen T, Nordentoft M, et al. Increased mortality among patients admitted with major psychiatric disorders: a registerbased study comparing mortality in unipolar depressive disorder, bipolar affective disorder, schizoaffective disorder, and schizophrenia. J Clin Psychiatry 2007;68:899-907.

2. Leucht S, Burkard T, Henderson J, et al. Physical illness and schizophrenia: a review of the literature. Acta Psychiatr Scand 2007;116:317-33.

3. Harris EC, Barraclough B. Excess mortality of mental disorder. $\mathrm{Br} \mathrm{J}$ Psychiatry 1998;173:11-53.

4. Joukamaa M, Heliovaara M, Knekt P, et al. Mental disorders and cause-specific mortality. Br J Psychiatry 2001;179:498-502.

5. Bushe CJ, Taylor M, Haukka J. Mortality in schizophrenia: a measurable clinical endpoint. J Psychopharmacol 2010;24 (4 Suppl):17-25

6. Druss BG, Zhao L, Von Esenwein S, et al. Understanding excess mortality in persons with mental illness: 17-year follow up of a nationally representative US survey. Med Care 2011;49:599-604.

7. Saha S, Chant D, McGrath J. A systematic review of mortality in schizophrenia: is the differential mortality gap worsening over time? Arch Gen Psychiatry 2007:64:1123-31.

8. Wahlbeck K, Westman J, Nordentoft M, et al. Outcomes of Nordic mental health systems: life expectancy of patients with mental disorders in Denmark, Finland and Sweden 1987-2006. Br J Psychiatry 2011;199:453-8.

9. Laursen TM, Munk-Olsen T, Agerbo E, et al. Somatic hospital contacts, invasive cardiac procedures, and mortality from heart disease in patients with severe mental disorder. Arch Gen Psychiatry 2009;66:713-20.

10. Lawrence D, Holman CD, Jablensky AV, et al. Excess cancer mortality in Western Australian psychiatric patients due to higher case fatality rates. Acta Psychiatr Scand 2000;101:382-8.
11. Lawrence D, Kisely S. Inequalities in healthcare provision for people with severe mental illness. J Psychopharmacol 2010;24(4 Suppl): $61-8$.

12. De Hert M, Correll CU, Bobes J, et al. Physical illness in patients with severe mental disorders. I. Prevalence, impact of medications and disparities in health care. World Psychiatry 2011:10:52-77.

13. Lawrence DM, Holman CD, Jablensky AV, et al. Death rate from ischaemic heart disease in Western Australian psychiatric patients 1980-1998. Br J Psychiatry 2003:182:31-6.

14. Kisely S, Campbell LA, Wang Y. Treatment of ischaemic heart disease and stroke in individuals with psychosis under universal healthcare. Br J Psychiatry 2009;195:545-50.

15. Hippisley-Cox J, Parker C, Coupland C, et al. Inequalities in the primary care of patients with coronary heart disease and serious mental health problems: a cross-sectional study. Heart 2007;93:1256-62.

16. Mitchell AJ, Lord O. Do deficits in cardiac care influence high mortality rates in schizophrenia? A systematic review and pooled analysis. J Psychopharmacol 2010;24(4 Suppl):69-80.

17. Druss BG, Rohrbaugh RM, Levinson CM, et al. Integrated medical care for patients with serious psychiatric illness: a randomized trial. Arch Gen Psychiatry 2001;58:861-8.

18. Desai MM, Rosenheck RA, Druss BG, et al. Mental disorders and quality of care among postacute myocardial infarction outpatients. J Nerv Ment Dis 2002;190:51-3.

19. Weiss AP, Henderson DC, Weilburg JB, et al. Treatment of cardiac risk factors among patients with schizophrenia and diabetes. Psychiatr Serv 2006;57:1145-52.

20. Mitchell AJ, Lawrence D. Revascularisation and mortality rates following acute coronary syndromes in people with severe mental illness: comparative meta-analysis. Br J Psychiatry 2011;198:434-41.

21. Druss BG, Bradford DW, Rosenheck RA, et al. Mental disorders and use of cardiovascular procedures after myocardial infarction. JAMA 2000;283:506-11.

22. Vuorenkoski L, Mladovsky P, Mossialos E. Finland: Health System Review. Health Systems in Transition. United Kingdom: WHO, 2008

23. Official Statistics of Finland (OSF). Causes of Death [e-publication]. ISSN=1799-5078. Helsinki: Statistics Finland. http://www.stat.fi/til/ ksyyt/ksyyt 2006-10-26 uut 001 en.html [referred: 9 Feb 2012]

24. Gissler M, Haukka J. Finnish health and social welfare registers in epidemiological research. Norsk Epidemiologi 2004;14:113-20.

25. Keskimäki I, Aro S. Accuracy of data on diagnosis, procedures and accidents in the Finnish Hospital Discharge Register. Int $J$ Health Sci 1991;2:15-21.

26. Pajunen $\mathrm{P}$, Koukkunen $\mathrm{H}$, Ketonen $\mathrm{M}$, et al. The validity of the Finnish hospital discharge register and causes of death register data on coronary heart disease. Eur J Cardiovasc Prev Rehabil 2005; $12: 132-7$

27. Perälä J, Suvisaari J, Saarni SI, et al. Lifetime prevalence of psychotic and bipolar I disorders in a general population. Arch Gen Psychiatry 2007;64:19-28.

28. Kieseppä T, Partonen T, Kaprio J, et al. Accuracy of register- and record-based bipolar I disorder diagnoses in Finland; a study of twins. Acta Neuropsychiatrica 2000;12:106-9.

29. Poikolainen K. Accuracy of hospital discharge data: five alcoholrelated diseases. Drug Alcohol Depend 1983;12:315-22.

30. Jones LE, Carney CP. Mental disorders and revascularization procedures in a commercially insured sample. Psychosom Med 2005;67:568-76.

31. Petersen LA, Normand SL, Druss BG, et al. Process of care and outcome after acute myocardial infarction for patients with mental illness in the VA health care system: are there disparities? Health Serv Res 2003;38:41-63.

32. Plomondon ME, Ho PM, Wang L, et al. Severe mental illness and mortality of hospitalized ACS patients in the VHA. BMC Health Serv Res 2007;7:146.

33. Kisely S, Smith M, Lawrence D, et al. Inequitable access for mentally ill patients to some medically necessary procedures. CMAJ 2007:176:779-84

34. Rusch N, Angermeyer MC, Corrigan PW. Mental illness stigma: concepts, consequences, and initiatives to reduce stigma. Eur Psychiatry 2005;20:529-39.

35. Aromaa E, Tolvanen A, Tuulari J, et al. Predictors of stigmatizing attitudes towards people with mental disorders in a general population in Finland. Nord J Psychiatry 2011;65:125-32.

36. Link BG, Phelan JC. Stigma and its public health implications. Lancet 2006;367:528-9.

37. Thornicroft G, Brohan E, Rose D, et al. Global pattern of experienced and anticipated discrimination against people with schizophrenia: a cross-sectional survey. Lancet 2009;373:408-15. 